\title{
Progresos educativos intergeneracionales: un análisis para Colombia
}

\author{
MORA RODRÍGUEZ, JHON JAMES \\ Facultad de Ciencias Administrativas y Económicas \\ Universidad Icesi (Colombia) \\ Correo electrónico: jjmora@icesi.edu.co \\ GONZÁLEZ TERÁN, ÁNGELA MARÍA \\ Facultad de Ciencias Administrativas y Económicas \\ Universidad Icesi (Colombia) \\ Correo electrónico: amgonzalez@icesi.edu.co
}

\begin{abstract}
RESUMEN
Este artículo analiza como las condiciones del entorno socioeconómico inciden sobre la movilidad en los niveles de educación secundaria, media y superior. Nuestro análisis se enfoca en la influencia del nivel educativo de los padres con el fin de evaluar la movilidad intergeneracional en educación. A partir de la estimación de un modelo logit de decisión secuencial dinámico nuestros resultados muestran que las condiciones del hogar inciden sobre el progreso en el sistema educativo ya que padres con niveles de educación superior son un fuerte apalancamiento para impulsar a sus hijos a través del sistema hasta la educación superior, siendo más fuerte esta influencia cuando se trata de la educación de la madre.
\end{abstract}

Palabras clave: movilidad educativa, modelos de elección discreta, progreso escolar.

Clasificación JEL: I24; I25; C21; C52; C55.

MSC2010: 91G70; 97B20; 62P20. 


\title{
Intergenerational educational progress: an analysis for Colombia
}

\begin{abstract}
This article discusses how the conditions of the socioeconomic environment affect mobility at the secondary, middle and upper levels of education. Our analysis focuses on the influence of parents' educational level to evaluate intergenerational mobility in education. Using a logit model of dynamic sequential decision our results show that the conditions of the home affect the progress in the education system since parents with higher education levels are a strong leverage to boost their children through the system even higher education, this influence being stronger when it comes to the mother education level.
\end{abstract}

Keywords: intergenerational mobility, discrete decision models, school progress.

JEL classification: I24; I25; C21; C52; C55.

MSC2010: 91G70; 97B20; 62P20.

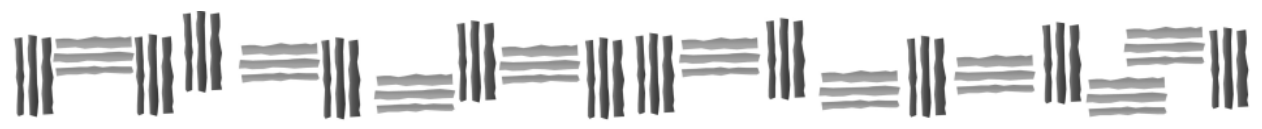




\section{Introducción.}

Colombia se enfrenta a dos retos cruciales en la educación: cerrar las brechas en términos de participación y mejorar la calidad de la educación (OCDE, 2016). Las desigualdades en educación tienen su origen incluso desde la temprana edad ya que muchos niños desfavorecidos nunca van a la escuela o no empiezan a tiempo o asisten a instituciones de menor calidad. Las diferencias en niveles de estudios alcanzados son abismales: la expectativa de vida escolar de los estudiantes con las peores condiciones de pobreza es de solo seis años, mientras que la expectativa de vida escolar es de 12 años para los más ricos y, solo el $9 \%$ en condiciones de pobreza se matricula en educación superior, en comparación con el $53 \%$ de los pertenecientes a las familias más acaudaladas.

Colombia es un país diverso y multiétnico, geográfica y territorialmente complejo. Estas características hacen particularmente exigente el camino del país hacia la equidad (PND, 2015). En general, aunque las condiciones de desigualdad del país han mejorado en los últimos años, la posición relativa que ocupa frente a otros países en vía de desarrollo del mismo continente continúa siendo muy baja. Situaciones como las grandes brechas regionales, la diferencia climática de cada una de las regiones, diferencias entre géneros y etnias, la distribución de las tierras y la distribución de los ingresos son algunos de los factores que contribuyen al incremento en la desigualdad.

La desigualdad afecta en gran medida el acceso a las oportunidades de los individuos en diversas áreas y una de ellas es la educación básica obligatoria que, incluso en algunos casos, no es ni siquiera una opción. Por lo tanto, como establece Becker (1964) ante las condiciones de desigualdad de un país que afecten especialmente al acceso a la educación se requerirá de una fuerte política de intervención estatal, para suplir las barreras que enfrenta la población más vulnerable.

La relevancia de analizar la movilidad intergeneracional en educación se hace más fuerte cuando el país presenta fallas en los mercados financiero y laboral, ya que se acentúan las desigualdades de ingresos entre los individuos frente a las posibilidades de acceso a la educación (Mediavilla \& Calero, 2010; Chau, 2012). En este sentido, este artículo busca analizar las condiciones dentro del entorno familiar del estudiante que afectan positiva y negativamente su progreso a través de los diferentes niveles educativos. El análisis aquí realizado parte de analizar cómo influye la educación de los padres sobre cada una de las decisiones de avanzar o no al siguiente nivel escolar, modelando la decisión de forma secuencial, observando diferencias a lo largo del tiempo a través de la incorporación de las cohortes.

El artículo se encuentra dividido en siete secciones, incluida la introducción. En la segunda sección se presenta una revisión de la literatura sobre movilidad educativa. En la tercera sección, se realiza un breve análisis del sistema educativo colombiano. En la cuarta sección se describe la metodología de un modelo econométrico logit secuencial. En la quinta sección, se presenta la base de datos y se muestran los resultados de una matriz de transición educativa no condicionada. En la sexta sección, aparecen los principales resultados y en la séptima sección se encuentran las conclusiones.

\section{La educación y la movilidad intergeneracional.}

Los estudios sobre el efecto de la educación sobre la movilidad intergeneracional analizan, en términos generales, cómo la educación de los padres incide sobre las elecciones de los hijos. Es así como uno de los primeros estudios sobre movilidad intergeneracional en educación fue el artículo de Lillard y Willis (1994), quienes exploran la relación entre la educación de padres e hijos, con datos longitudinales para Malasia. Los autores encuentran que las decisiones de 
educación de los padres sobre los hijos siguen siendo fuertes y que es mayor el efecto de la madre que el efecto del padre.

Cameron y Heckman (2001) estiman un modelo dinámico de asistencia escolar para investigar las causas de las disparidades étnico-raciales, observando el progreso para los últimos tres a cuatro años de colegio hasta la entrada a la universidad para afrodescendientes, hispanos y blancos. Los autores encuentran que la trayectoria de los padres y el ambiente familiar determinan las diferencias étnicas y raciales en el progreso escolar en lugar de las restricciones de crédito. Iannelli y Paterson (2007) miden las diferencias entre cohortes del efecto de la educación de la generación anterior sobre la movilidad entre estatus socioeconómicos. El estudio lo realizan para Escocia con datos de 2001 y sus resultados muestran que los hogares de clase media transmiten ventajas a sus hijos mientras que los hogares de clase baja perpetúan las desventajas y que la movilidad se ve fuertemente condicionada por la educación de los padres.

Ben-Halima, Chusseau, y Hellier (2014) analizan el impacto de la educación de los padres y el ingreso de estos sobre la asistencia escolar de sus niños y su desempeño en el examen de estado. Usan datos de la encuesta de formación de la calificación profesional para seis olas tomadas para intervalos de seis años desde 1964 hasta 2003 y encuentran que a mayor nivel de educación de los padres mayor es el desempeño en la prueba de estado, mientras que menores niveles de educación reducen la probabilidad de obtener mejores puntuaciones.

Kwenda, Ntuli, y Gwatidzo (2015) analizan las sendas de transmisión intergeneracional en educación entre los afrodescendientes de Sudáfrica. Con datos de cohortes de nacimiento desde 1954 hasta 1993, encuentran una disminución en la transmisión intergeneracional de educación para las últimas cuatro décadas. De igual forma encuentran, que los hijos de las familias más pobres presentan una relación más fuerte con el nivel educativo de sus padres.

Huebener (2015) analiza cómo afecta la aversión al riesgo de los padres en la transmisión intergeneracional de ingresos y educación. Utilizando datos de panel de la encuesta socio económica alemana de padres e hijos desde 1984 a 2012. Y encuentra que hijos con padres que tienen una mayor aversión al riesgo tienen menor movilidad intergeneracional tanto en educación como en ingresos.

Qin, Wang, y Zhuang (2016) estiman el impacto de la transmisión intergeneracional de capital humano en la movilidad de ingresos en China. Usando datos desde 1989 hasta 2009 encuentran que el capital humano se transmite de forma directa (genéticamente) e indirecta (a través del ambiente familiar y los ingresos invertidos en capital humano).

Finalmente, Heckman y Raut (2016), analizan la decisión de inversión en las opciones de preescolar de padres altruistas Los autores concluyen que la inversión en preescolar tiene efectos significativos sobre las habilidades cognitivas del joven y que están asociadas a los ingresos futuros y los resultados escolares.

Para el caso colombiano, Baltazar, Astudillo, y Malaver (2003) analizan la relación entre la baja escolaridad y los hogares con menores recursos para la ciudad de Bogotá. Sus resultados muestran que la movilidad es ascendente para los deciles de ingresos más altos y descendentes para los más bajos.

Cartagena (2005) analiza la movilidad educativa entre generaciones desde 1915 hasta 2003, creando un índice de movilidad ascendente y construyendo una serie de rentabilidad de la educación. Sus resultados muestran un aumento de la educación entre generaciones y señalan una disminución en el ritmo de crecimiento de la movilidad intergeneracional para los últimos treinta años. 
Viáfara, Estacio, y González (2010) analizan la movilidad educativa intergeneracional para las clases medias afrodescendientes en las ciudades de Bogotá, Cali y el agregado de las trece áreas metropolitanas y encuentran que este grupo enfrenta una mayor inmovilidad que las clases medias no afrodescendientes, siendo el efecto mayor en la ciudad de Bogotá.

Bonilla (2010) analiza la movilidad intergeneracional en educación desde una perspectiva regional, con el fin de analizar en cuales ciudades y regiones la educación de los individuos depende menos de la de sus padres. Sus resultados muestran que existen diferencias en los niveles de movilidad entre las zonas centrales y la periferia, siendo baja para los segundos y alta en los primeros.

Viáfara y López (2011) analizan los factores que influyen en la baja movilidad educativa de los afrodescendientes. Ellos encuentran que la baja movilidad no solo se debe a la condición de pobreza del hogar ni a la menor educación de sus padres, sino también a la presencia de desventajas acumulativas derivadas de la discriminación por raza y sexo. A través de un modelo logístico ordenado comprueban su hipótesis para datos de la encuesta CIDSE-IRD (1998) para la ciudad de Cali.

Morales (2012) analiza las diferencias por género en la movilidad intergeneracional educativa a través de las cohortes de nacimiento para el área metropolitana de Cali. Utilizando la Encuesta de Calidad de Vida de 2003 y modelos de decisión discreta encuentra que la movilidad educativa aumenta para las mujeres y disminuye en los hombres.

González, Mora, y Albert (2013) analizan la demanda de educación universitaria en Colombia con datos de las encuestas de hogares del DANE encontrando que el género, el nivel educativo de los padres, la situación laboral de éstos, la composición familiar y el ingreso son determinantes en la decisión de estudiar más. González, Mora y Cuadros (2014) analizan el efecto de las características familiares sobre el acceso a la educación postobligatoria en Colombia. Utilizando un modelo probit ordenado generalizado con los datos de la GEIH para el periodo 2008 a 2012, encuentran que las características como la educación de los padres y su situación laboral tienen una fuerte relación con la demanda de educación no obligatoria, existiendo diferencias entre géneros y niveles de ingresos.

Por su parte, Moyano y Galvis (2014) identifican los determinantes de la brecha educativa para los adolescentes en Colombia. Los autores consideran que los adolescentes provenientes de hogares con padres más educados y con mayores ingresos per cápita son los que presentan las menores brechas educativas.

Galvis y Roca (2014) analizan la movilidad social en Colombia a nivel regional. Para hacerlo calculan la movilidad promedio entre padres e hijos. Sus conclusiones dejan ver que existe una significativa transmisión del logro educativo entre generaciones.

Finalmente, Ayala (2015) analiza si los hogares que solo cuentan con madres cabeza de familia se enfrentan a mayores restricciones con respecto a los hogares con tienen dos padres, en la transmisión intergeneracional de educación e ingresos. Con los datos de la Encuesta Nacional de Calidad de Vida (ENCV) estiman un modelo logit ordenado para los niveles educativos de los hijos y encuentran que se requiere que al menos uno de los padres haya alcanzado altos niveles educativos para que los hijos alcancen estudios universitarios.

\section{La educación en Colombia.}

La educación en Colombia se encuentra dividida entre obligatoria y no obligatoria. Por mandato constitucional la educación es obligatoria solo entre los cinco y quince años, que equivalen a noveno grado. La educación media (10-13) y la educación superior (técnica, tecnológica y universitaria) no son obligatorias y, por lo tanto, son decisiones propias del individuo y su familia. 
Y, es esta clase de separación entre niveles obligatorios y post obligatorios lo que refuerza la correlación que existe entre las características familiares y el acceso a la educación (González, Mora, \& Cuadros, 2014).

La OCDE (2016) muestra que Colombia presenta bajas tasas de asistencia escolar. Esto es debido en parte a una deficiente transición entre niveles, altas tasas de deserción escolar y un número significativo de niños que no ingresan al sistema educativo. Y, aunque la tasa de deserción desde preescolar hasta la educación secundaria se ha reducido a más de la mitad entre 2002 y 2013 en Colombia, las tasas de cobertura bruta han aumentado. La proporción de niños en edad de cursar primaria que no están estudiando ha aumentado del 4\% en 2000 al 9\% en 2010 (OCDE, 2016). Aproximadamente uno de cada cinco estudiantes en Colombia no continúa estudiando después de la primaria. La proporción de jóvenes en edad de cursar educación básica secundaria que no están estudiando ha disminuido del 15\% en 2005 al 9\% en 2013.

La financiación de la educación colombiana es altamente centralizada. La mayor parte de los recursos para la educación desde transición hasta el último año de educación media son transferidos por el Ministerio de Educación Nacional (MEN) a los departamentos, los municipios y las escuelas y colegios por medio del sistema general de participaciones (SGP). Entre los años 2002 y 2013, la cuota de los gastos totales en educación del SGP se redujo notablemente, pasando del $80 \%$ al $60 \%$, y la proporción de gastos directos por parte del gobierno nacional pasó a ser casi el doble, del 9\% al 17\%. Asimismo, los gastos de las entidades territoriales certificados (ETC) provenientes de sus propios recursos aumentaron. Sin embargo, representaron solamente el 13\% de los gastos totales en el año 2013. Si bien la relación entre gastos totales (públicos y privados) por estudiantes de educación básica primaria y secundaria y el PIB (20\% y 21\%, respectivamente) es cercana al promedio de los países de la OCDE (23\% y 26\%, respectivamente), en términos absolutos, los gastos por estudiante son muy bajos, de manera que los recursos privados desempeñan un papel más importante que en la mayor parte de los países miembros de la OCDE (OCDE, 2016).

Por su parte, la educación básica secundaria sigue siendo el eslabón más débil del sistema educativo colombiano, con una tasa de deserción anual del 4.5\%, superior a la de la educación primaria (3.2 \%) y educación media (3.1\%) (UNICEF, 2016). Solo el 30\% de los jóvenes hace la transición de la escuela o colegio a la educación superior y de estos, muchos desertarán antes de terminar. Las tasas de deserción anual en educación superior varían entre el $10.4 \%$ en las universidades y el 22.2\% en las instituciones técnicas y tecnológicas (PND, 2015). Las constantes tasas de deserción y la débil transición indican que Colombia tiene uno de los porcentajes más altos de jóvenes entre los 15 y los 19 años de edad que no están estudiando: un 36\% en total (OCDE, 2016). Además, el 19\% de los jóvenes entre los 15 y 19 años ni estudia, ni trabaja, ni recibe educación, doblando la proporción promedio de los países de la OCDE (Mora, Caicedo y González, 2017). Muchos obstáculos limitan el acceso de los jóvenes a la educación, entre otros, la falta de oportunidades educativas, la pobreza, la presión por empezar a trabajar, el conflicto y la violencia (OCDE, 2016).

El análisis del sistema educativo colombiano muestra que la intervención estatal aún no es lo suficientemente fuerte para solventar las fallas del mercado, permitiendo el crecimiento de las brechas en educación. Por tal motivo, se hace imperante verificar cuáles son los factores que más repercusiones tienen en el avance dentro del sistema escolar. Esto con el fin de reajustar el portafolio de inversiones en política pública en educación hacia aquellos aspectos cuya intervención genere el mayor impacto sobre el progreso escolar. El presente documento está orientado bajo esta idea, explora la movilidad intergeneracional en educación en Colombia buscando los factores que determinan las decisiones de continuar a un nivel mayor de educación. 


\section{Metodología.}

Con el fin de analizar el progreso de los jóvenes a lo largo de los niveles educativos proponemos un modelo de decisión secuencial, donde la posibilidad de avanzar al periodo siguiente, en un nivel educativo, dependerá de la decisión en el periodo anterior. Esto nos permitirá, periodo a periodo, analizar el efecto de los padres en las decisiones educativas de sus hijos.

La Figura 1 presenta la elección de educación utilizando el árbol de las decisiones secuenciales para analizar el progreso escolar en Colombia. Esta figura muestra cómo el proceso de elección escolar es secuencial, a diferencia de González, Mora y Cuadros (2014) quienes consideran que la elección de demanda puede ser analizada a través de un probit ordenado.

Figura 1. Árbol de las decisiones secuenciales de progreso escolar en Colombia.

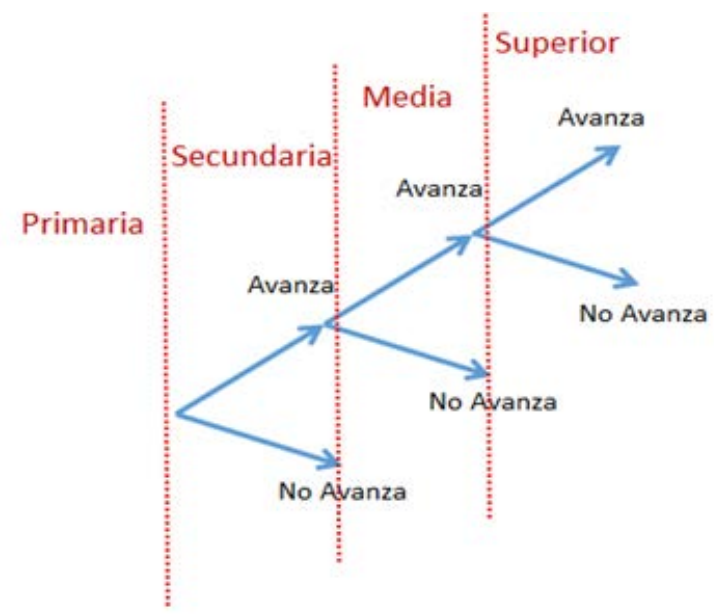

Fuente: Elaboración propia.

En particular, supondremos que el individuo que se encuentra en el sistema escolar, que existen $N$ individuos y que $Y_{i}$ es la variable de decisión con $S$ posibles opciones ordenadas $\{s=1, \ldots, J\}$. A continuación, sea $s$ el grado de estudio, $D_{s}=1$ si una persona completa el grado $s$ y $D_{s}=0$ en caso contrario. El nivel de estudio alcanzado en $s$ es determinado por el individuo en el grado anterior $s$ - 1 . Siendo $X_{s}=x_{s}$ los regresores que determinan la probabilidad de avanzar del grado $s-1$ al grado $s$, dada por

$$
\operatorname{Pr}\left(D_{s}=1 \mid X_{s}=x_{s}, D_{s-1}=1\right)=P_{s-1, s}\left(x_{s}\right)
$$

El evento $D_{s-1}=0$ implica que un individuo abandona el sistema educativo en el grado $s-1$, lo que significa que se excluyen los resultados de los eventos sucesivos $D_{s}=1$ cuando $D_{s-1}=0$. Siguiendo a Cameron y Heckman (1998) estas probabilidades modelan el proceso de transición escolar, que se convierte en un proceso markoviano completamente especificado cuando se asume una condición inicial $D_{1}$, mediante el uso de una probabilidad logística se plantea que la posibilidad de transcender de un nivel educativo al siguiente es:

$$
P_{s-1, s} x_{s}^{\prime}=\frac{\exp \left(x_{s}^{\prime} \beta_{s}\right)}{1+\exp \left(x_{s}^{\prime} \beta_{s}\right)}
$$

De tal forma que para cada nivel educativo $\left(S_{i(t)}\right)$ la probabilidad de un individuo $i$ de continuar al siguiente nivel está dada por:

$$
S_{i(t)}=1\left[x_{i(t)}^{\prime} \beta_{i(t)}+\epsilon_{i(t)}>0 \mid S_{i(t-1)}>0\right]
$$


Donde $\epsilon_{i(t)}$ se distribuye valor extremo tipo I y $x_{i(t)}$ es el vector de las características del individuo, las características del hogar y las características de los padres.

\section{Datos analizados.}

Para el análisis de cómo influye el contexto familiar en el progreso escolar se usaron datos de la Gran Encuesta Integrada de Hogares (GEIH) del Departamento Administrativo Nacional de Estadísticas (DANE). Se tomaron ocho años de encuestas desde 2008 hasta 2016.

El uso de este gran número de encuestas de corte transversal se fundamenta en la oportunidad que brinda para la creación de 8 cohortes de nacimiento que permiten observar el avance dinámico entre niveles de educación para los individuos de la cohorte.

La tabla 1 muestra los datos después de unir todas las etapas de la GEIH. En términos generales la proporción de hombres y mujeres es similar. La edad promedio es de 25 años, el $43 \%$ de los individuos tiene internet, pero solo el 36\% de estos posee computador. El 16\% de los individuos tuene un carro mientras que el $8 \%$ vive en la zona rural. El ingreso promedio del hogar es $\$ 908.909$ pesos colombianos (USD 300.5). El numero promedio de personas por hogar es de 5.

Tabla 1. Estadísticas descriptivas de las variables independientes del modelo secuencial.

\begin{tabular}{lrrrrr}
\hline Variable & Obs. & Media & Desv. Est. & Mínimo & Máximo \\
\hline Sexo & 116558 & 0.51 & 0.50 & 0 & 1 \\
Edad & 116558 & 25.17 & 10.20 & 11 & 80 \\
Internet & 116508 & 0.43 & 0.50 & 0 & 1 \\
Computador & 116508 & 0.36 & 0.48 & 0 & 1 \\
Carro & 116508 & 0.16 & 0.36 & 0 & 1 \\
Rural & 116558 & 0.08 & 0.28 & 0 & 1 \\
Ingresos hogar (log) & 106413 & 13.72 & 0.96 & 8.23 & 19.93 \\
Personas por hogar & 116558 & 5.02 & 2.10 & 2 & 25 \\
Nivel educativo (padre) & 71344 & 3.06 & 1.50 & 1 & 7 \\
Nivel educativo (madre) & 110600 & 2.99 & 1.42 & 1 & 7 \\
Departamentos & 116558 & 37.54 & 23.23 & 5 & 76 \\
Cohortes & 107639 & 6.35 & 1.88 & 1 & 8 \\
\hline
\end{tabular}

Fuente: Elaboración propia a partir de GEIH-DANE.

Los datos se analizan a partir de seis niveles educativos: ninguno, preescolar, primaria, secundaria, media y superior (Tabla 2). En la tabla 2 se observa cómo, a partir de la cohorte de los individuos nacidos entre 1972 y 1976, aumenta el porcentaje que completa la educación superior siendo este porcentaje superior al 20\%. A partir de la cohorte de individuos nacidos en 1977 las proporciones de individuos que terminaron el bachillerato y completaron la educación superior eran mayoría frente a los individuos que solo alcanzaban primaria y secundaria. Por ejemplo, para los individuos nacidos entre 1982 y 1986 el 34\% terminaron la educación media y el $47 \%$ se graduaron de educación superior. Solo el $18 \%$ de ellos se quedaron en los niveles inferiores de educación (primaria y secundaria) 
Tabla 2. Porcentaje de individuos en cada nivel educativo por rangos de año de nacimientos (cohortes).

\begin{tabular}{ccccccc}
\hline $\begin{array}{c}\text { Años de } \\
\text { Nacimiento }\end{array}$ & Ninguno & Primaria (1-5) & Secundaria (6-9) & Media (10-13) & Superior & Total \\
\hline $1917-1946$ & 20.2 & 56.4 & 11.2 & 6.2 & 5.9 & 100.0 \\
$1947-1951$ & 12.5 & 50.0 & 14.1 & 11.5 & 11.9 & 100.0 \\
$1952-1956$ & 8.1 & 44.6 & 17.6 & 15.3 & 14.4 & 100.0 \\
$1957-1961$ & 7.1 & 39.8 & 19.2 & 19.7 & 14.2 & 100.0 \\
$1962-1966$ & 5.5 & 36.3 & 21.5 & 23.9 & 12.8 & 100.0 \\
$1967-1971$ & 4.0 & 34.8 & 20.6 & 25.7 & 14.9 & 100.0 \\
$1972-1976$ & 3.6 & 27.1 & 16.0 & 27.8 & 25.5 & 100.0 \\
$1977-1981$ & 1.4 & 12.0 & 11.5 & 32.6 & 42.6 & 100.0 \\
$1982-1986$ & 1.1 & 8.2 & 9.8 & 34.2 & 46.7 & 100.0 \\
$1987-1991$ & $0-6$ & 7.3 & 12.8 & 37.7 & 41.6 & 100.0 \\
$1992-1996$ & 0.9 & 9.6 & 23.5 & 34.3 & 31.7 & 100.0 \\
Total & 4.8 & 26.3 & 16.3 & 26.4 & 26.2 & 100.0 \\
\hline \multicolumn{7}{c}{ Fuente: Elaboración propia a partir de GEIH-DANE. } \\
\end{tabular}

A continuación, se procedió a analizar la transición entre los niveles educativos de los individuos entre las generaciones. Para esto se calculó la proporción de individuos que cambiaron o permanecieron en cada uno de los niveles educativos, de una generación a otra, es decir, de padres a hijos y de hijos a nietos, tomando todos los individuos observados con las encuestas de 2008 a 2016. La tabla 2 muestra el resultado de estas transiciones.

Tabla 3. Matrices de transición no condicionada (periodos 2008-2016).

\begin{tabular}{ccccccc}
\hline & Nivel Educativo & Ninguno & Primaria (1-5) & Secundaria (6-9) & Media (10-13 & Superior \\
\hline Padre/Hijo & Ninguno & 7.2 & 31.13 & 21.91 & 28.64 & 11.13 \\
& Primaria (1-5) & 1.12 & 14.33 & 18.94 & 39.1 & 26.51 \\
& Secundaria (6-9) & 0.33 & 3.61 & 14.42 & 39.5 & 42.13 \\
& Media (10-13) & 0.09 & 1.61 & 6.95 & 33.94 & 57.41 \\
& Superior & 0 & 0.44 & 2.62 & 15.15 & 81.79 \\
\cline { 2 - 7 } Hijo/Nieto & Total & 1.17 & 9.83 & 14.22 & 34.44 & 40.33 \\
& Ninguno & 2.22 & 42.22 & 31.11 & 22.22 & 2.22 \\
& Primaria (1-5) & 0.91 & 16.97 & 22.73 & 40 & 19.39 \\
& Secundaria (6-9) & 0.36 & 5.07 & 18.12 & 38.41 & 38.04 \\
& Media (10-13) & 0.85 & 3.39 & 10.17 & 44.63 & 40.96 \\
& Superior & 0 & 1.64 & 6.15 & 20.49 & 71.72 \\
\hline \hline
\end{tabular}

Fuente: Elaboración propia a partir de GEIH-DANE.

En la tabla 3 se puede observar que cuando el padre no tiene ningún nivel educativo la educación del hijo estará concentrada en los niveles de primaria, secundaria y media, con mayor probabilidad en primaria (31.13\%) y media (28.6\%). Cuando el padre alcanza al menos la primaria, aumenta forma significativa la probabilidad de que el hijo alcance la educación media (39\%). No obstante, si el padre tiene educación superior la probabilidad de que el hijo supere la educación media y termine la educación superior es bastante alta (82\%). 
Este comportamiento es consistente tanto en las relaciones de padre a hijo como de hijo a nieto, siendo más marcadas en la relación de hijo a nieto para los niveles más bajos de educación del padre y más fuerte el apalancamiento cuando el padre tiene educación superior, si se trata de la relación de padre a hijo. Como conclusión previa de estos cálculos se puede esperar que si los padres tienen bajos niveles educativos esto reduce la probabilidad de que sus hijos alcancen un nivel de educación superior.

\section{Resultados del modelo.}

Para la estimación del modelo dinámico de decisión secuencial se utilizó como variable dependiente el grado de movilidad intergeneracional en educación, que corresponde al nivel educativo del padre y al nivel educativo de la madre. Como variables de control se utilizaron el sexo, la edad, el departamento y la cohorte de nacimiento del estudiante. También se controló por características del hogar, como el número de personas que lo componen y si se trata de un hogar rural. Igualmente, se incorporó la tenencia de ciertos bienes como el coche (vehículo propio), el ordenador y el acceso a internet, las condiciones de estudio y transporte escolar y el ingreso percibido por el hogar. Los resultados del modelo se pueden observar en la tabla 4.

Tabla 4. Efectos marginales del modelo secuencial para los periodos de 2008 al 2016.

\begin{tabular}{|c|c|c|c|}
\hline & Secundaria & Media & Superior \\
\hline Hombre & $\begin{array}{l}-0.0487 * * * \\
(0.00205)\end{array}$ & $\begin{array}{l}-0.0697 * * * \\
(0.00305)\end{array}$ & $\begin{array}{l}-0.101^{* * *} \\
(0.00442)\end{array}$ \\
\hline Edad & $\begin{array}{l}0.000130 \\
(0.000375)\end{array}$ & $\begin{array}{l}0.0444 * * * \\
(0.000598)\end{array}$ & $\begin{array}{l}0.0192 * * * \\
(0.000846)\end{array}$ \\
\hline Internet & $\begin{array}{l}0.0137 * * * \\
(0.00259)\end{array}$ & $\begin{array}{l}0.0322 * * * \\
(0.00346)\end{array}$ & $\begin{array}{l}0.0629 * * * \\
(0.00555)\end{array}$ \\
\hline Computador & $\begin{array}{l}0.0495^{* * *} \\
(0.00260)\end{array}$ & $\begin{array}{l}0.0805^{* * *} \\
(0.00383)\end{array}$ & $\begin{array}{l}0.129 * * * \\
(0.00584)\end{array}$ \\
\hline Carro & $\begin{array}{l}0.0289 * * * \\
(0.00365)\end{array}$ & $\begin{array}{l}0.0173^{* * *} \\
(0.00432)\end{array}$ & $\begin{array}{l}0.0713 * * * \\
(0.00620)\end{array}$ \\
\hline Rural & $\begin{array}{l}-0.0759 * * * \\
(0.00355)\end{array}$ & $\begin{array}{l}-0.0224 * * * \\
(0.00614)\end{array}$ & $\begin{array}{l}-0.100 * * * \\
(0.0105)\end{array}$ \\
\hline Ingresos Hogar (log) & $\begin{array}{l}0.0207^{* * *} \\
(0.00124)\end{array}$ & $\begin{array}{l}0.0194 * * * \\
(0.00209)\end{array}$ & $\begin{array}{l}0.0500^{* * *} \\
(0.00300)\end{array}$ \\
\hline Personas por hogar & $\begin{array}{l}-0.00827 * * * \\
(0.000457)\end{array}$ & $\begin{array}{l}-0.0193 * * * \\
(0.000897)\end{array}$ & $\begin{array}{l}-0.0374^{* * *} \\
0.00133\end{array}$ \\
\hline Primaria (Padre) & $\begin{array}{l}0.0380^{* * * *} \\
(0.00378)\end{array}$ & $\begin{array}{l}0.0659 * * * \\
(0.00821)\end{array}$ & $\begin{array}{l}0.0528 * * * \\
(0.0124)\end{array}$ \\
\hline Secundaria (Padre) & $\begin{array}{l}0.0671^{* * *} \\
(0.00461)\end{array}$ & $\begin{array}{l}0.0875^{* * *} \\
(0.00880)\end{array}$ & $\begin{array}{l}0.0907^{* * *} \\
(0.0133)\end{array}$ \\
\hline Media (Padre) & $\begin{array}{l}0.0729 * * * \\
(0.00513)\end{array}$ & $\begin{array}{l}0.106 * * * \\
(0.00886)\end{array}$ & $\begin{array}{l}0.127 * * * \\
(0.0135)\end{array}$ \\
\hline Técnico/Tecnológico (Padre) & $\begin{array}{l}0.0852 * * * \\
(0.00933)\end{array}$ & $\begin{array}{l}0.117 * * * \\
(0.0109)\end{array}$ & $\begin{array}{l}0.180 * * * \\
(0.0166)\end{array}$ \\
\hline
\end{tabular}




\begin{tabular}{|c|c|c|c|}
\hline Universitario (Padre) & $\begin{array}{l}0.0844^{* * *} \\
(0.00892)\end{array}$ & $\begin{array}{l}0.0898^{* * *} \\
(0.0103)\end{array}$ & $\begin{array}{l}0.181^{* * *} \\
(0.0158)\end{array}$ \\
\hline Posgrado (Padre) & $\begin{array}{l}0.0717^{* * *} \\
(0.0202)\end{array}$ & $\begin{array}{l}0.0936^{* * *} \\
(0.0130)\end{array}$ & $\begin{array}{l}0.178^{* * *} \\
(0.0207)\end{array}$ \\
\hline Primaria (Madre) & $\begin{array}{l}0.0534 * * * \\
(0.00445)\end{array}$ & $\begin{array}{l}0.0692 * * * \\
(0.00912)\end{array}$ & $\begin{array}{l}0.0747 * * * \\
(0.0137)\end{array}$ \\
\hline Secundaria (Madre) & $\begin{array}{l}0.0930 * * * \\
(0.00510)\end{array}$ & $\begin{array}{l}0.0974 * * * \\
(0.00958)\end{array}$ & $\begin{array}{l}0.135^{* * *} \\
(0.0144)\end{array}$ \\
\hline Media (Madre) & $\begin{array}{l}0.112^{* * *} \\
(0.00520)\end{array}$ & $\begin{array}{l}0.136^{* * *} \\
(0.00965)\end{array}$ & $\begin{array}{l}0.177 * * * \\
(0.0147)\end{array}$ \\
\hline Técnico/Tecnológico (Madre) & $\begin{array}{l}0.111^{* * *} \\
(0.00777)\end{array}$ & $\begin{array}{l}0.130 * * * \\
(0.0111)\end{array}$ & $\begin{array}{l}0.212^{* * *} \\
(0.0173)\end{array}$ \\
\hline Universitario (Madre) & $\begin{array}{l}0.122 * * * \\
(0.00770)\end{array}$ & $\begin{array}{l}0.102^{* * *} \\
(0.0113)\end{array}$ & $\begin{array}{l}0.224^{* * *} \\
(0.0175)\end{array}$ \\
\hline Posgrado (Madre) & $\begin{array}{l}0.133 * * * \\
(0.00987)\end{array}$ & $\begin{array}{l}0.116 * * * \\
(0.0142)\end{array}$ & $\begin{array}{l}0.225^{* * *} \\
(0.0229)\end{array}$ \\
\hline Atlántico & $\begin{array}{l}0.0270 * * * \\
(0.00616)\end{array}$ & $\begin{array}{l}0.0502 * * * \\
(0.00882)\end{array}$ & $\begin{array}{l}-0.0191 \\
(0.0124)\end{array}$ \\
\hline Bolívar & $\begin{array}{l}0.0257 * * * \\
(0.00656)\end{array}$ & $\begin{array}{l}0.0455^{* * *} \\
(0.00924)\end{array}$ & $\begin{array}{l}0.0798^{* * *} \\
(0.0133)\end{array}$ \\
\hline Boyacá & $\begin{array}{l}0.0145^{*} \\
(0.00696)\end{array}$ & $\begin{array}{l}0.0577 * * * \\
(0.00984)\end{array}$ & $\begin{array}{l}0.0933^{* * *} \\
(0.0140)\end{array}$ \\
\hline Caldas & $\begin{array}{l}-0.0108 \\
(0.00729)\end{array}$ & $\begin{array}{l}0.0155 \\
(0.0101)\end{array}$ & $\begin{array}{l}-0.0338 * \\
(0.0132)\end{array}$ \\
\hline Caquetá & $\begin{array}{l}-0.0244 * * * \\
(0.00739)\end{array}$ & $\begin{array}{l}0.00529 \\
(0.0107)\end{array}$ & $\begin{array}{l}0.0229 \\
(0.0166)\end{array}$ \\
\hline Cauca & $\begin{array}{l}0.00855 \\
(0.00653)\end{array}$ & $\begin{array}{l}0.0386 * * * \\
(0.00969)\end{array}$ & $\begin{array}{l}0.105^{* * *} \\
(0.0135)\end{array}$ \\
\hline Cesar & $\begin{array}{l}0.0166^{*} \\
(0.00665)\end{array}$ & $\begin{array}{l}0.0532 * * * \\
(0.00925)\end{array}$ & $\begin{array}{l}0.0659 * * * \\
(0.0139)\end{array}$ \\
\hline Córdoba & $\begin{array}{l}0.0233 * * * \\
(0.00626)\end{array}$ & $\begin{array}{l}0.0604 * * * \\
(0.00929)\end{array}$ & $\begin{array}{l}-0.0121 \\
(0.0136)\end{array}$ \\
\hline Cundinamarca & $\begin{array}{l}0.0174 * \\
(0.00688)\end{array}$ & $\begin{array}{l}0.0246^{* *} \\
(0.00883)\end{array}$ & $\begin{array}{l}0.0455^{* * *} \\
(0.0122)\end{array}$ \\
\hline Chocó & $\begin{array}{l}0.0214 * * \\
(0.00726)\end{array}$ & $\begin{array}{l}0.0456^{* * *} \\
(0.0109)\end{array}$ & $\begin{array}{l}0.0660^{* * *} \\
(0.0178)\end{array}$ \\
\hline Huila & $\begin{array}{l}-0.0140 * \\
(0.00704)\end{array}$ & $\begin{array}{l}0.00184 \\
(0.0100)\end{array}$ & $\begin{array}{l}0.0405^{* *} \\
(0.0139)\end{array}$ \\
\hline La Guajira & $\begin{array}{l}0.00744 \\
(0.00684)\end{array}$ & $\begin{array}{l}0.0390 * * * \\
(0.00942)\end{array}$ & $\begin{array}{l}0.0839 * * * \\
(0.0143)\end{array}$ \\
\hline
\end{tabular}




\begin{tabular}{|c|c|c|c|}
\hline Magdalena & $\begin{array}{l}0.0214^{* *} \\
(0.00731)\end{array}$ & $\begin{array}{l}0.0452 * * * \\
(0.00971)\end{array}$ & $\begin{array}{l}-0.0545^{* * *} \\
(0.0139)\end{array}$ \\
\hline Meta & $\begin{array}{l}0.0101 \\
(0.00746)\end{array}$ & $\begin{array}{l}0.0120 \\
(0.0103)\end{array}$ & $\begin{array}{l}-0.125^{* * *} \\
(0.0143)\end{array}$ \\
\hline Nariño & $\begin{array}{l}-0.0119 \\
(0.00627)\end{array}$ & $\begin{array}{l}0.0360 * * * \\
(0.00964)\end{array}$ & $\begin{array}{l}0.0140 \\
(0.0132)\end{array}$ \\
\hline Norte de Santander & $\begin{array}{l}-0.0342 * * * \\
(0.00692)\end{array}$ & $\begin{array}{l}0.0267 * * \\
(0.0100)\end{array}$ & $\begin{array}{l}0.0241 \\
(0.0143)\end{array}$ \\
\hline Quindío & $\begin{array}{l}0.00730 \\
(0.00713)\end{array}$ & $\begin{array}{l}0.00738 \\
(0.0102)\end{array}$ & $\begin{array}{l}-0.0136 \\
(0.0143)\end{array}$ \\
\hline Risaralda & $\begin{array}{l}-0.0170 * \\
(0.00842)\end{array}$ & $\begin{array}{l}-0.0132 \\
(0.0108)\end{array}$ & $\begin{array}{l}-0.0774 * * * \\
(0.0147)\end{array}$ \\
\hline Santander & $\begin{array}{l}-0.0141 \\
(0.00720)\end{array}$ & $\begin{array}{l}0.0225 * \\
(0.00986)\end{array}$ & $\begin{array}{l}-0.00128 \\
(0.0133)\end{array}$ \\
\hline Sucre & $\begin{array}{l}0.0225 * * * \\
(0.00582)\end{array}$ & $\begin{array}{l}0.0455 * * * \\
(0.00893)\end{array}$ & $\begin{array}{l}0.0742 * * * \\
(0.0132)\end{array}$ \\
\hline Tolima & $\begin{array}{l}0.00653 \\
(0.00725)\end{array}$ & $\begin{array}{l}0.0348 * * * \\
(0.0101)\end{array}$ & $\begin{array}{l}0.0111 \\
(0.0139)\end{array}$ \\
\hline Valle del Cauca & $\begin{array}{l}0.0125 \\
(0.00697)\end{array}$ & $\begin{array}{l}-0.00449 \\
(0.00981)\end{array}$ & $\begin{array}{l}-0.117 * * * \\
(0.0129)\end{array}$ \\
\hline 1961-1965 & $\begin{array}{l}0.0937 * * * \\
(0.0201)\end{array}$ & $\begin{array}{l}0.0265 * * * \\
(0.00251)\end{array}$ & $\begin{array}{l}0.0854 * * * \\
(0.0205)\end{array}$ \\
\hline $1966-1970$ & $\begin{array}{l}0.0879 * * * \\
(0.0203)\end{array}$ & $\begin{array}{l}0.0818 * * * \\
(0.00414)\end{array}$ & $\begin{array}{l}0.136^{* * *} \\
(0.0185)\end{array}$ \\
\hline 1971-1975 & $\begin{array}{l}0.131^{* * *} \\
(0.0228)\end{array}$ & $\begin{array}{l}0.191^{* * *} \\
(0.00664)\end{array}$ & $\begin{array}{l}0.229 * * * \\
(0.0177)\end{array}$ \\
\hline $1976-1980$ & $\begin{array}{l}0.155 * * * \\
(0.0252)\end{array}$ & $\begin{array}{l}0.410^{* * *} \\
(0.00868)\end{array}$ & $\begin{array}{l}0.332 * * * \\
(0.0172)\end{array}$ \\
\hline 1981-1985 & $\begin{array}{l}0.179 * * * \\
(0.0270)\end{array}$ & $\begin{array}{l}0.630 * * * \\
(0.00644)\end{array}$ & $\begin{array}{l}0.424 * * * \\
(0.0180)\end{array}$ \\
\hline 1986-1990 & $\begin{array}{l}0.194 * * * \\
(0.0285)\end{array}$ & $\begin{array}{l}0.814^{* * *} \\
(0.00330)\end{array}$ & $\begin{array}{l}0.487 * * * \\
(0.0197)\end{array}$ \\
\hline 1991-1995 & $\begin{array}{l}0.197 * * * \\
(0.0296)\end{array}$ & $\begin{array}{l}0.759 * * * \\
(0.00243)\end{array}$ & $\begin{array}{l}0.426 * * * \\
(0.0217)\end{array}$ \\
\hline
\end{tabular}

Errores estándar entre paréntesis. $* \mathrm{p}<0.05, * * \mathrm{p}<0.01, * * * \mathrm{p}<0.001$

Fuente: Elaboración propia a partir de la GEIH (2008-2016).

La tabla 4 muestra que las variables son estadísticamente significativas en el caso de las variables de edad, ingresos, educación de los padres y cohortes. Algunas variables regionales correspondientes a departamentos no fueron estadísticamente significativas. 
Los resultados de la estimación muestran que los hombres tienen menor probabilidad de pasar de un nivel educativo al otro y que estas probabilidades se van reduciendo en los niveles de educación superior. De esta forma, ser hombre disminuye la probabilidad de pasar a secundaria en 5 puntos porcentuales frente a la probabilidad de hacerlo que tuviera si fuese mujer. La probabilidad de superar la educación media se reduce aún más (en 7 puntos) pero el mayor efecto por género se presenta cuando se analiza la probabilidad de alcanzar la educación superior ya que ésta se reduce en 10 puntos porcentuales cuando el estudiante es hombre, esto es, más del doble que lo que se reduce la de alcanzar secundaria.

La edad del estudiante tiene un efecto positivo para el progreso escolar, disminuyendo la magnitud de su impacto para el último nivel educativo. Con cada año adicional el estudiante incrementa su probabilidad de superar el nivel educativo en 4 puntos porcentuales para educación media y en 2 puntos para educación superior. Esto puede implicar que las edades demasiado bajas podrían afectar el progreso escolar.

Las condiciones del hogar afectan de forma significativa el progreso escolar ya que poseer un ordenador, internet o coche incrementa la probabilidad de alcanzar un nivel educativo superior como es el universitario. Es así como la existencia de internet en el hogar tiene como efecto el cambio de 1 punto porcentual en primaria a 6 puntos porcentuales sobre la probabilidad de alcanzar un nivel de educación superior. El efecto de poseer un ordenador es más fuerte todavía, pasando de menos de 5 a 12 puntos porcentuales en la transición a secundaria hasta la transición por la educación superior. De lo anterior, se puede deducir que la tecnología hoy en día se ha convertido en un factor relevante para el estudio de los jóvenes, en especial la capacidad de acceder a un ordenador con internet. Por otro lado, el poseer transporte propio como coche también afecta positivamente el progreso del estudiante, teniendo su mayor efecto en el paso de la educación superior.

La ubicación geográfica del hogar es importante ya que si se trata de un hogar rural la probabilidad de pasar al siguiente nivel educativo se reduce en todos los casos. Sin embargo, su efecto es mayor para el salto entre primaria y secundaria (7 puntos porcentuales) y cuando se decide alcanzar el nivel de educación superior (10 puntos), mientras que el efecto que tiene sobre la probabilidad de pasar a educación media es tan solo de 2 puntos porcentuales, frente a la situación en la que el hogar es urbano.

Los ingresos del hogar muestran que a mayor ingreso mayor es el nivel de educación, de esta forma un incremento de un $1 \%$ en los ingresos del hogar incrementa la probabilidad de llegar a la educación universitaria en 5 puntos porcentuales. Para la educación secundaria y media el cambio en la probabilidad de avanzar es de alrededor de 2 puntos porcentuales.

Un factor que genera efectos negativos sobre la probabilidad de pasar al siguiente nivel educativo es el número de personas que se encuentran en el hogar, dado que su efecto es mayor a medida que el estudiante avanza en su educación. Una persona adicional en el hogar disminuye la probabilidad de avanzar a la educación secundaria en 0.9 puntos porcentuales, a la educación media en 2 puntos porcentuales y en 4 puntos la de avanzar a educación superior.

Las características de los padres siguen siendo influyentes en las decisiones de progreso escolar. Por parte del padre se puede apreciar que el nivel educativo del mismo es significativo para el avance en todos los niveles de educación de su hijo. Es decir que, según sea el nivel educativo del padre, se afecta la decisión de progreso escolar del hijo. Esto sucede también para el caso de la madre, en el que todos los niveles de educación son significativos e incluso tienen mayor efecto que el del padre, en especial para la cola superior. De esta forma, cuando el padre ha alcanzado solamente la primaria la probabilidad de que el hijo alcancé secundaria se incrementa en 4 puntos porcentuales con respecto a la situación en la que el padre no tiene ningún nivel educativo. Pero el incremento en esta probabilidad disminuye para la transición a educación media y superior siendo sólo de 7 y 5 puntos porcentuales respectivamente. El efecto de la 
educación de la madre es el doble para el paso a la educación secundaria y similar al del padre con educación primaria para los otros dos niveles. De igual forma, se puede observar que si el padre posee educación secundaria la probabilidad del hijo de alcanzar secundaria se incrementa en 7 puntos porcentuales con respecto a un padre sin educación y en 9 puntos porcentuales cuando se trata de la probabilidad de alcanzar la educación media y superior. En el caso del efecto de la educación de la madre, ésta tiene un impacto mayor que la del padre para cada transición, siendo siempre una y media veces más grandes que el del padre con el mismo nivel educativo: secundaria. Cuando el padre ha alcanzado la educación media, esto es, que ha completado toda la educación preuniversitaria (educación obligatoria), la probabilidad de que sus hijos alcancen al menos su mismo nivel se incrementa en 10 puntos porcentuales con respecto a la probabilidad que tendrían los hijos de un padre no educado. La madre con educación media, por su parte, tiene un mayor impacto que el padre con el mismo nivel de educación ya que el efecto crece con cada nivel educativo, pasando de 11 hasta 18 puntos porcentuales adicionales. Cuando ambos padres tienen educación superior, las probabilidades de que sus hijos alcancen niveles educativos altos son mayores: la probabilidad que los hijos de individuos con educación superior pasen a secundaria es de 8 puntos porcentuales, que alcancen media es de 9 y que logren niveles como los de sus padres de educación superior es de 18, siempre con respecto a los hijos de los padres menos educados.

Geográficamente, existen diferencias en torno a alcanzar un determinado nivel de educación con respecto al departamento base. En Bolívar, Boyacá, Cauca, Cesar, Cundinamarca, Choco, Huila, La Guajira y Sucre la probabilidad de alcanzar educación universitaria es mayor que la probabilidad de alcanzar educación primaria y secundaria. En departamentos como Caldas, Meta y Valle del Cauca la probabilidad de llegar al nivel de educación universitario es inferior con respecto al departamento base.

Finalmente, la incorporación de cohortes muestra que existen diferencias para cada una de las cohortes de nacimiento. Las cohortes de antes de los 70's tenían menores probabilidades de llegar a tener educación secundaria que las cohortes después de los años 70’s. Para las cohortes más jóvenes el incremento en la educación media de alrededor de 20 puntos porcentuales por cohorte. El mayor salto se observa entre la cohorte de 1971-1975 a la cohorte de 1976-1980 donde la probabilidad de superar la educación media es más del doble entre éstas.

\section{Conclusiones.}

La movilidad intergeneracional en la educación ha sido considerada como uno de los principales mecanismos de transmisión de desigualdad en una sociedad. En países en vía de desarrollo como Colombia, las condiciones institucionales, culturales y del entorno socioeconómico de un joven aún tienen demasiada influencia sobre su progreso escolar, en especial en los niveles de educación no obligatoria. Es así como, este artículo analiza el peso de estas características del entorno familiar en las decisiones de progreso escolar de los estudiantes, concentrándose en la influencia de la educación de sus padres sobre sus hijos. De esta forma, los resultados aquí encontrados muestran que en efecto existen variables que tienen un gran impacto en el avance de los jóvenes a lo largo de los niveles educativos del sistema escolar colombiano.

Las condiciones del hogar afectan en mayor medida la probabilidad de alcanzar la educación superior. Esto quiere decir que la carencia de alguna de estas características hace más probable que el estudiante no avance más allá de educación media. El análisis por cohortes permite observar que con el tiempo las probabilidades de avanzar entre los niveles educativos se han ido incrementando, teniendo un fuerte cambio a partir de los años 70’s.

El nivel educativo alcanzado por los padres es importante a la hora de establecer la posibilidad de sus hijos de progresar en el sistema educativo. Padres que al menos hayan terminado la educación preuniversitaria tienen hijos con buenas probabilidades de alcanzar su mismo nivel educativo y de avanzar a la educación superior. No obstante, tener padres con 
educación superior es determinante para el estudiante, ya que aumenta la probabilidad de que se alcanzará la educación superior.

A pesar de los esfuerzos del gobierno por impulsar la educación en las zonas rurales, los resultados confirman que siguen presentándose diferencias con respecto a aquellos estudiantes que viven en zonas urbanas por factores potencialmente institucionales y culturales que permean las decisiones familiares de educación. Es menos probable que los estudiantes en las zonas rurales alcancen la educación secundaria, aunque una vez superado este nivel sus condiciones no tienen tanta influencia en su decisión de seguir a la educación media pero sí la tienen en la decisión de alcanzar la educación superior.

Es claro que el progreso en el sistema educativo es un proceso que aún depende mucho de las características de los padres, en particular de su nivel de estudios. Es por ello que políticas de mejoramiento y expansión de la oferta educativa y subsidios a las familias de escasos recursos, aumenta la probabilidad de alcanzar niveles de educación superior. Por lo tanto, la inversión en educación de los jóvenes de hoy es una forma de apalancar el progreso educativo de sus hijos y de los hijos de sus hijos.

Finalmente, aunque es claro que se hace necesario un impulso al género masculino en el avance dentro del sistema educativo (debido a que presenta menores probabilidades de lograrlo que el género femenino), también es claro que apoyar a las mujeres en su educación es una política mucho más efectiva para impulsar a los hijos de esta generación en el mejoramiento del nivel educativo. Políticas públicas que fomenten la educación superior en cualquiera de sus formas (técnico, tecnológico y universitario) son fuertes mecanismos para garantizar mayores niveles de educación en la población colombiana de hoy y de mañana.

\section{Referencias}

Ayala, C. (2015). Movilidad educativa intergeneracional en mujeres cabeza de familia para las áreas metropolitanas de Barranquilla, Bogotá, Cali y Medellín (Tesis Doctoral, Universidad del Valle). Descargado de http://hdl.handle.net/10893/ 7936

Baltazar, E.N., Astudillo, S.G., \& Malaver, C. A. (2003). Movilidad social y transmisión de la pobreza en Bogotá. Economía y Desarrollo, 2(2), 119-156.

Becker, G.S. (1964). Human capital. A theoretical and empirical analysis, with special reference to education (Vol 4) (No. 3 Pt. 2). National Bureau of Economic Research.

Ben-Halima, B., Chusseau, N., \& Hellier, J. (2014). Skill premia and intergenerational education mobility: The French case. Economics of Education Review, 39, 50-64.

Bonilla, L. (2010). Movilidad intergeneracional en educación en las ciudades y regiones de Colombia. Revista de Economía del Rosario, 13(2), 191-233.

Cameron, S., \& Heckman, J. (1998). Life Cycle Schooling and Dynamic Selection Bias: Models and Evidence for Five Cohorts of American Males. Journal of Political Economy, 106(2), 262-333.

Cameron, S. V., \& Heckman, J. J. (2001). The Dynamics of Educational Attainment for Black, Hispanic, and White Males. Journal of Political Economy, 109(3), 455-499.

Cartagena, K. (2005). Movilidad intergeneracional en Colombia. Revista ESPE, 1(51), 208-261. 
Chau, T.W. (2012). Intergenerational income mobility revisited. Estimation with an income dynamic model with heterogeneous age profile. Economics Letters, 117(3), 770-773.

Galvis, L., \& Roca, A.M. (2014). Aspectos regionales de la movilidad social y la igualdad de oportunidades en Colombia. Documentos de trabajo sobre Economía Regional, 55. Descargado de http://www.banrep.gov.co/ publicaciones/pub\{_\}ec\{_\}reg4.htm.

González, C.G., Mora, J.J., y Albert, C. (2013). Determinantes de la demanda de educación universitaria en Colombia. Revista de Economía Institucional, 15, 169-194.

González, C.G., Mora, J.J., \& Cuadros, A.F. (2014). Oportunidades educativas y características familiares en Colombia: un análisis por cohortes. Revista de Economía del Rosario, 17(1), 157-187.

Heckman, J. J., \& Raut, L. K. (2016). Intergenerational Long Term Effects of Preschool Structural Estimates from a Discrete Dynamic Programming Model. NBER Working Paper, 191(19077), 164-175.

Huebener, M. (2015). The role of paternal risk attitudes in long-run education outcomes and intergenerational mobility. Economics of Education Review, 47, 64-79.

Iannelli, C., \& Paterson, L. (2007). Education and social mobility in Scotland. Research in Social Stratification and Mobility, 25(3), 219-232.

Kwenda, P., Ntuli, M., \& Gwatidzo, T. (2015). Temporal developments in intergenerational transmission of education: Case for black South Africans. Research in Social Stratification and Mobility, 42, 96-113.

Lillard, L.A., \& Willis, R.J. (1994). Intergenerational Educational Mobility: Effects of Family and State in Malaysia. The Journal of Human Resources, 29(4), 1126-1166.

Mediavilla, M., \& Calero, J. (2010). Education Mobility in Latin America. A Study of Six Countries. Revista Española de Educación Comparada (REEC), 16, 287-303.

Mora, J.J, Caicedo, C., \& González, C.G. (2017). La Duración del Desempleo de los Jóvenes y los “ninis” en Cali”. Revista de Economía Institucional, 19(37), 167-184.

Morales, A. (2012). Movilidad educativa intergeneracional por género en el Área Metropolitana de Cali ¿existen diferencias por cohortes? (Tesis de grado). Universidad del Valle. Descargado de http://bibliotecadigital.univalle.edu.co/bitstream/10893/3708/4/ CB0460622.pdf

Moyano, M., \& Galvis, L. (2014). ¿Oportunidades para el futuro?: la movilidad social de los adolescentes en Colombia. Documentos de trabajo sobre economía regional y urbana, 11. Banco de la República-Economía Regional.

OCDE, Organización para la Cooperación del Desarrollo Económico (2016). La educación en Colombia. París: Organización para la Cooperación y el Desarrollo Económico, Ed.

PND, Plan Nacional de Desarrollo. (2015). Plan Nacional de Desarrollo, Todos Por un Nuevo País 2014-2018, 53(9). 
Qin, X., Wang, T., \& Zhuang, C.C. (2016). Intergenerational transfer of human capital and its impact on income mobility: Evidence from China. China Economic Review, 38, 306-321.

UNICEF, Fondo de las Naciones Unidas para la Infancia. (2016). Una oportunidad para cada niño. Nueva York: División de Comunicaciones de UNICEF.

Viáfara, C., Estacio, A., \& González, L. (2010). Condición étnico-racial, género y movilidad social en Bogotá, Cali y el agregado de las trece áreas metropolitanas en Colombia: un análisis descriptivo y econométrico. Revista Sociedad y Economía, 18, 113-136.

Viáfara C., \& López, A. (2011). Efectos de la raza y el sexo en el logro educativo y estatus ocupacional en el primer empleo en la ciudad de Cali Colombia. Revista Sociedad y Economía, 11, 66-95. 